International Conference on Renewable Energies and Power Quality (ICREPQ'11)

Las Palmas de Gran Canaria (Spain), 13th to 15th April, 2011

\title{
Study on Flow Characteristic of Lubricant in the Main Shaft of Transmission in Commercial Vehicle
}

\author{
Ji Hun Yun ${ }^{1}$, Jeong Se Suh ${ }^{2 *}$, Chung Seob $\mathrm{Yi}^{2}$, Chul Ki Song ${ }^{2}$, In Guk Jeong ${ }^{1}$ and Joong Hwan Park ${ }^{3}$ \\ ${ }^{1}$ Graduate School of Mechanical Engineering, \\ Gyeongsang National University, Jinju, Gyeongnam 660-701, Korea, \\ e-mail: hoon0570@gmail.com, cdlsrnr@gmail.com \\ ${ }^{2}$ School of Mechanical and Aerospace Engineering, \\ Gyeongsang National University, Jinju, Gyeongnam 660-701, Korea \\ Phone number: +82-55-751-5312, e-mail: jssuh@gnu.ac.kr, cs_yi@ hotmail.com, cksong@gnu.ac.kr \\ ${ }^{3}$ S\&T Dynamics, \\ Oe-dong 853-5, Seongsan-gu, Changwon, Gyeongnam 642-020, Korea \\ Phone number: +82-55-751-5312, e-mail: parkjhw@ hisntd.com
}

\begin{abstract}
In this study, numerical simulation has been performed to investigate the flow characteristic of lubricant in the main shaft of transmission in commercial vehicle. It was confirmed that the mass flow rate of lubricating oil depends on the length of branch and the rotating rate of shaft. As a result, it could be verified that the discharged flow rate is considerably influenced by the length of the branch of main shaft. This study suggests the optimal design data for the lubrication system of the transmission in commercial vehicle.
\end{abstract}

\section{Key words}

Numerical analysis, Transmission, Commercial vehicle, Main shaft, Lubricant flow

\section{Introduction}

Recently, due to high performance and capacity engine for not only passenger cars but commercial vehicles, the required transmission power has especially been increasing for commercial vehicles. The powerful lubricant system is required for supplying lubricant oil to the transmission of commercial vehicle to reduce the occurrence of wear and heat in the gear box under the condition of high speed and heavy load.

Moreover, recently, a consumer's demand for green cars has been increased in the automobile industry to resolve the environmental pollution problem. The effort for improving the efficiency of engine combustion and power transferring in transmission during car operation has also been emphasized in the commercial vehicles.

Especially, the legalistic establishment changing carbon dioxide regulation with auto-maker is in progress for the object to application in Europe in 2012 and the legislation of fuel efficiency reinforcement is also promoted in U.S.A. So, in situation under the policy of reducing carbon dioxide related to global warming and the rising high oil prices, the development of environment-friendly commercial vehicles and the improvement of fuel efficiency are an essential task for automobile industry to survive and the technical development for high fuel efficiency transmission goes forward actively.

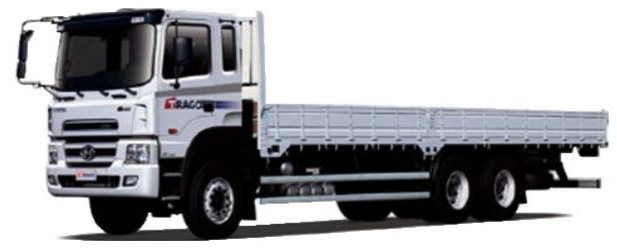

(a) cargo

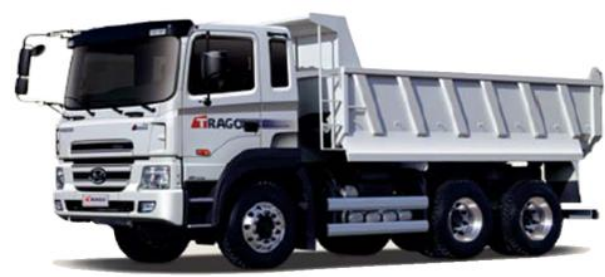

(b) dump

Fig. 1 Types of commercial vehicle using the transmission system studied in this work

The multi-range transmission can be the one of the technical developments for high fuel efficiency commercial vehicles transmission. But the performance of the transmission system is reduced by increasing of volume and mass of multi-range transmission system. To reduce this performance loss of the transmission system, the modified reformation of transmission system should be made by the miniaturization and reducing weight of 
the transmission parts. But the miniaturization and reducing weight of the transmission parts yields the performance reducing of the cooling and lubrication system that is regarded as the most important factor in transmission system. The operation of transmission necessarily produces the bearing wear and frictional heat generation at the joining part, which affects to the durability of transmission. Therefore, the flow rate of transmission lubricant controls the wearing of bearing from friction and minimizes the frictional heat generation, which can reduce the viscosity drop by the elevated temperature. ${ }^{[1-3]}$

In this study, numerical simulation has been made to investigate the flow characteristic of lubricant for transmission system. Additionally, the effect of the length of branch on the distribution of the lubricant will be primarily investigated in the inner main shaft of transmission

\section{Manual Transmission Lubrication System}

The structure of main shaft in transmission of commercial vehicle is shown in Fig.2. The transmission system has rearwheel drive type, nine steps and multi-range gear box. The transmission fluid is supplied from the oil pump to mainshaft through the bearing retainer as shown in Fig.3.

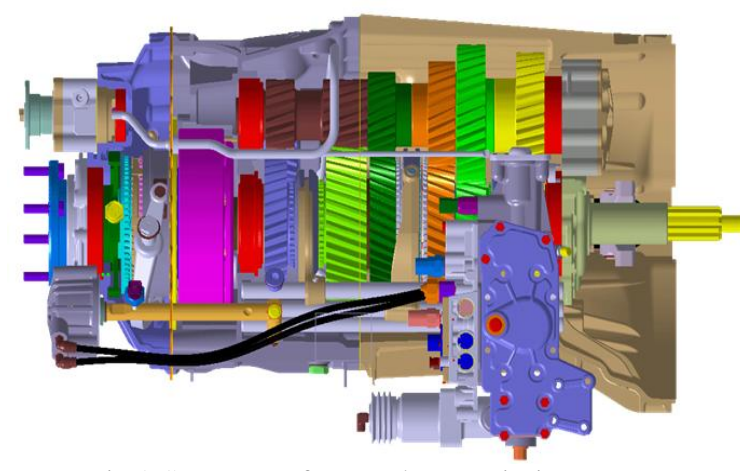

Fig.2 Structure of manual transmission system

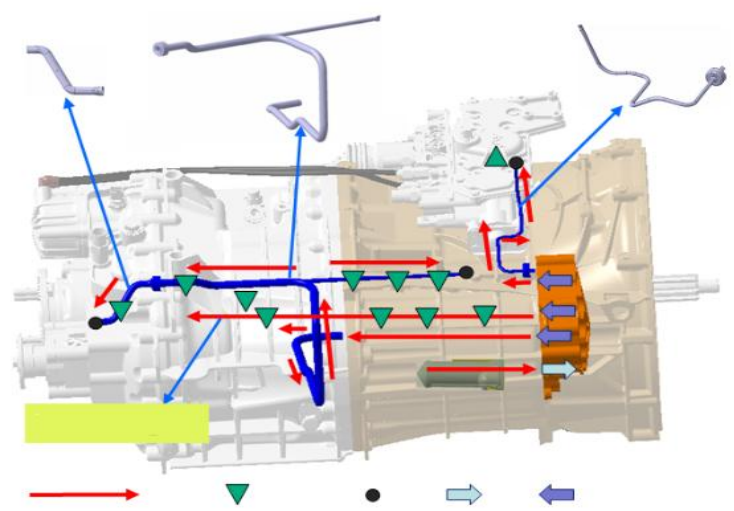

Fig.3 Lubrication channel of transmission system

Transmission lubricant used in this study is the viscosity coefficient of $0.0106 \mathrm{~Pa}\left(32^{\circ} \mathrm{C}\right)$ in case of $80 \mathrm{~W} / 90$ on the SAE viscosity level. The shape of oil hole discharging the lubricant is schematically depicted in Fig. 4 and the inner diameter of main shaft, the oil branch diameter of main shaft and the distance to branch have been presented in Table I.
Table I.- Geometric parameters of a main shaft

\begin{tabular}{l|c|c|c|c|c|c}
\hline \hline $\begin{array}{l}\text { Branch } \\
\text { No. }\end{array}$ & 1 & 2 & 3 & 4 & 5 & 6 \\
\hline Distance & 121.1 & 188.7 & 260.2 & 329.9 & 428.1 & 509.2 \\
\hline Diameter & 4 & 4 & 4 & 4 & 4 & 4 \\
\hline
\end{tabular}

Shaft inner diameter : $12 \mathrm{~mm}$
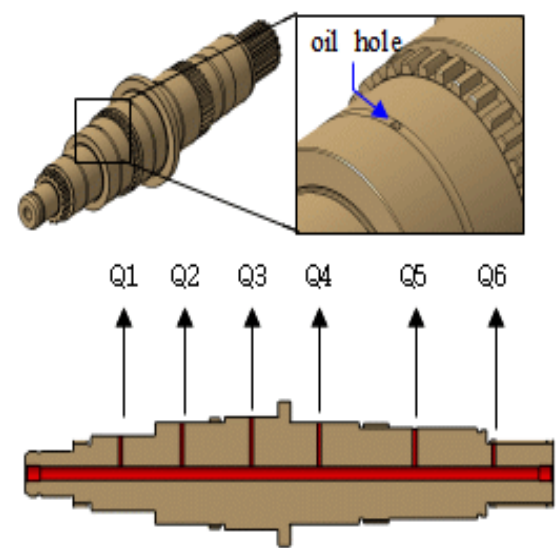

$\rightarrow$ Flow direction

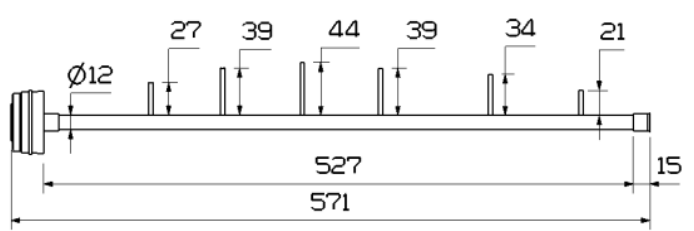

Fig. 4 Main shaft of transmission

\section{Analysis model and boundary conditions}

\section{A. Governing Equation}

In this study, the governing equation specifying the flow of lubrication fluid in the commercial vehicle is assumed to 3D steady turbulent flow. In case of transmission fluid, it can be assumed to turbulent flow because of its high viscosity and the turbulent flow caused by the complex geometric flow channel from oil pump to lubrication system. The equations of continuity, momentum, turbulent flow energy and the dissipation energy of turbulent flow motion related with lubricant flow can be considered. The density and viscosity of lubricant fluid is assumed to constant. The two equation kinetic models of k- $\varepsilon$ are used for turbulent flow field.

Continuity equation

$$
\frac{\partial}{\partial x_{i}}\left(\rho u_{i}\right)=0
$$

Momentum equation

$$
\frac{\partial}{\partial x_{j}}\left(\rho u_{j} u_{i}\right)=\frac{\partial P}{\partial x_{i}}+\frac{\partial \tau_{i j}}{\partial x_{i}}+S_{u}
$$


Turbulence kinetic energy equation

$$
\frac{\partial\left(\rho u_{j} k\right)}{\partial x_{j}}=\frac{\partial}{\partial x_{j}}\left(\mu+\frac{\mu_{t}}{\sigma_{k}}\right) \frac{\partial k}{\partial x_{j}}+\mu_{t} G-\sigma \varepsilon
$$

Turbulence kinetic disappearance equation

$$
\frac{\partial\left(\rho u_{j} \varepsilon\right)}{\partial x_{j}}=\frac{\partial}{\partial x_{j}}\left(\mu+\frac{\mu_{t}}{\sigma_{\varepsilon}}\right) \frac{\partial \varepsilon}{\partial x_{j}}+\frac{\varepsilon}{k}\left(C_{1} \mu_{t} G-C_{2} \rho \varepsilon\right)
$$

where

$$
\begin{gathered}
\mu_{t}=\frac{C \mu \rho k^{2}}{\varepsilon} \\
\tau_{t}=-\left(\mu+\mu_{t}\right)\left(\frac{\partial u_{i}}{\partial x_{j}}+\frac{\partial u_{j}}{\partial x_{i}}\right) \\
S_{u}=-\frac{2}{3}\left(\mu+\mu_{t}\right) \frac{\partial}{\partial x_{i}}\left(\frac{\partial u_{i}}{\partial x_{i}}\right) \\
\mu_{t} G=\mu_{t}\left(\frac{\partial u_{i}}{\partial x_{j}}+\frac{\partial u_{j}}{\partial x_{i}}\right) \frac{\partial u_{i}}{\partial x_{j}}-\frac{2}{3}\left(\rho k+\frac{\partial u_{i}}{\partial x_{i}}\right) \frac{\partial u_{j}}{\partial x_{j}} \\
C_{1}=1.44, \quad C_{2}=1.92, \quad C_{\mu}=0.09 \quad \sigma_{k}=1.0 \quad \sigma_{\varepsilon}=0.9 \\
\text { B. Boundary Conditions }
\end{gathered}
$$

\section{B. Boundary Conditions}

The boundary condition at the main shaft inlet is given by the Eq. (9) to find theoretical discharged flux in lubrication pump as shown in Fig. 5.

$$
Q_{t h}=N \times V_{t h} \times(A-B) \times t, \quad V_{t h}=Z \times(A-B) \times t
$$

where $V_{t h}$ is the exclusive volume, $N$ the number of rotations of oil pump, $Z$ the number of lobe in the inner rotor and $t$ the thickness of the rotor.

Fig. 6 represents the theoretical flux, discharged from the oil pump and flowed into the main shaft inlet. Since the oil pump is installed at count shaft, the flux of final engine rotating number is calculated by gear ratio (31/27) to obtain the flux of engine rotating number which is installed at main shaft. Because of numerical analysis about main shaft, the air pressure was given as boundary conditions at each branch.

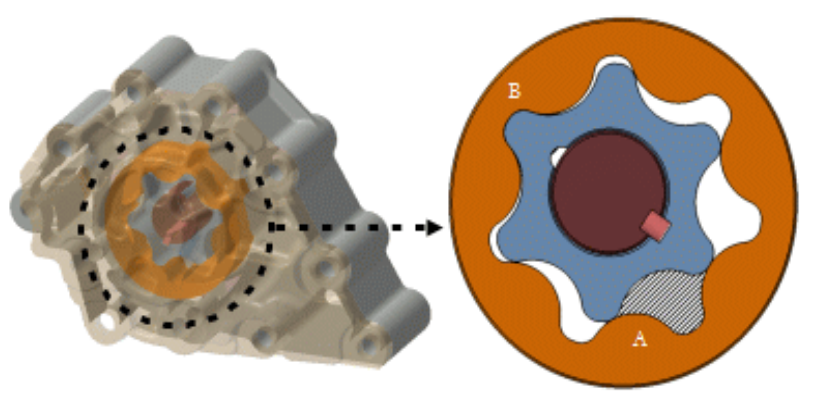

Fig. 5 Oil pump and rotor section

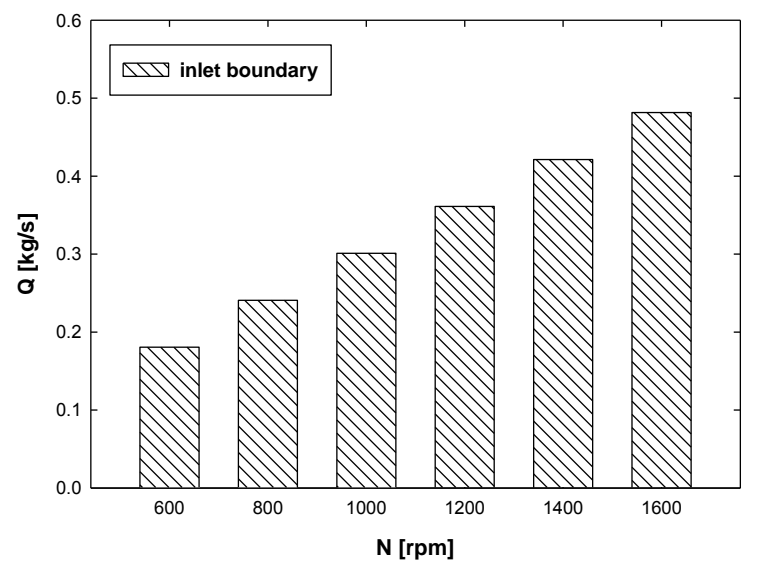

Fig. 6 Theory mass flow rate at main shaft inlet

\section{Validation of Mesh System}

The mesh shape of control volume is mainly adapted in polyhedral type in this numerical analysis. For the improvement of numerical accuracy in the computational result, the numerical results are preliminary compared by changing the cell size of computational domain for the operating condition of the minimum and maximum rotating number of main shaft. In Fig. 7, it can be found that the numerical results for the cell size of $2 \mathrm{~mm}$ are in a good agreement with those of the cell size of $1 \mathrm{~mm}$.

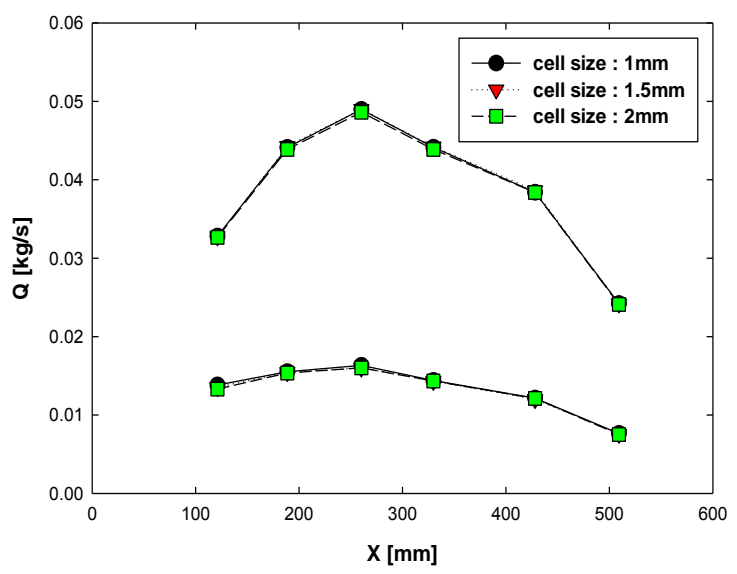

Fig. 7 Validation of mesh size

Therefore, the cell size of $2 \mathrm{~mm}$ will be mainly adapted to save the computing time in this study and the total number of the cells is approximately 516,100 for the computational domain.

\section{Results and Discussion}

For the engine rotating speed of main shaft from 600rpm to commercial rotating speed of $1600 \mathrm{rpm}$, the mass flow rate distribution of discharged transmission fluid is shown in Fig. 8.

As shown in Fig. 8, the discharging mass flow rate of transmission fluid linearly increases with increasing engine rotating rate. The discharging mass flow rate of transmission fluid at the branch is due to pressure rise from the rotation rate of main shaft. 


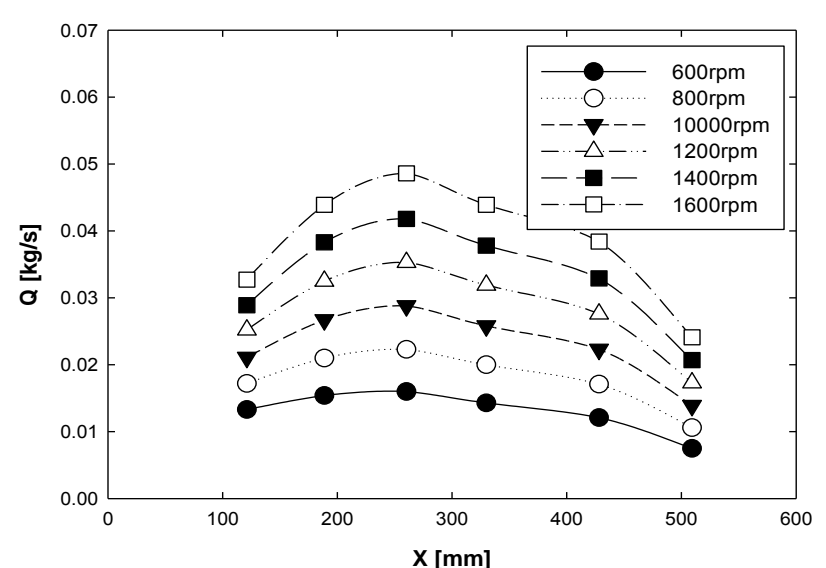

Fig. 8 Distributions of mass flow rate at branch
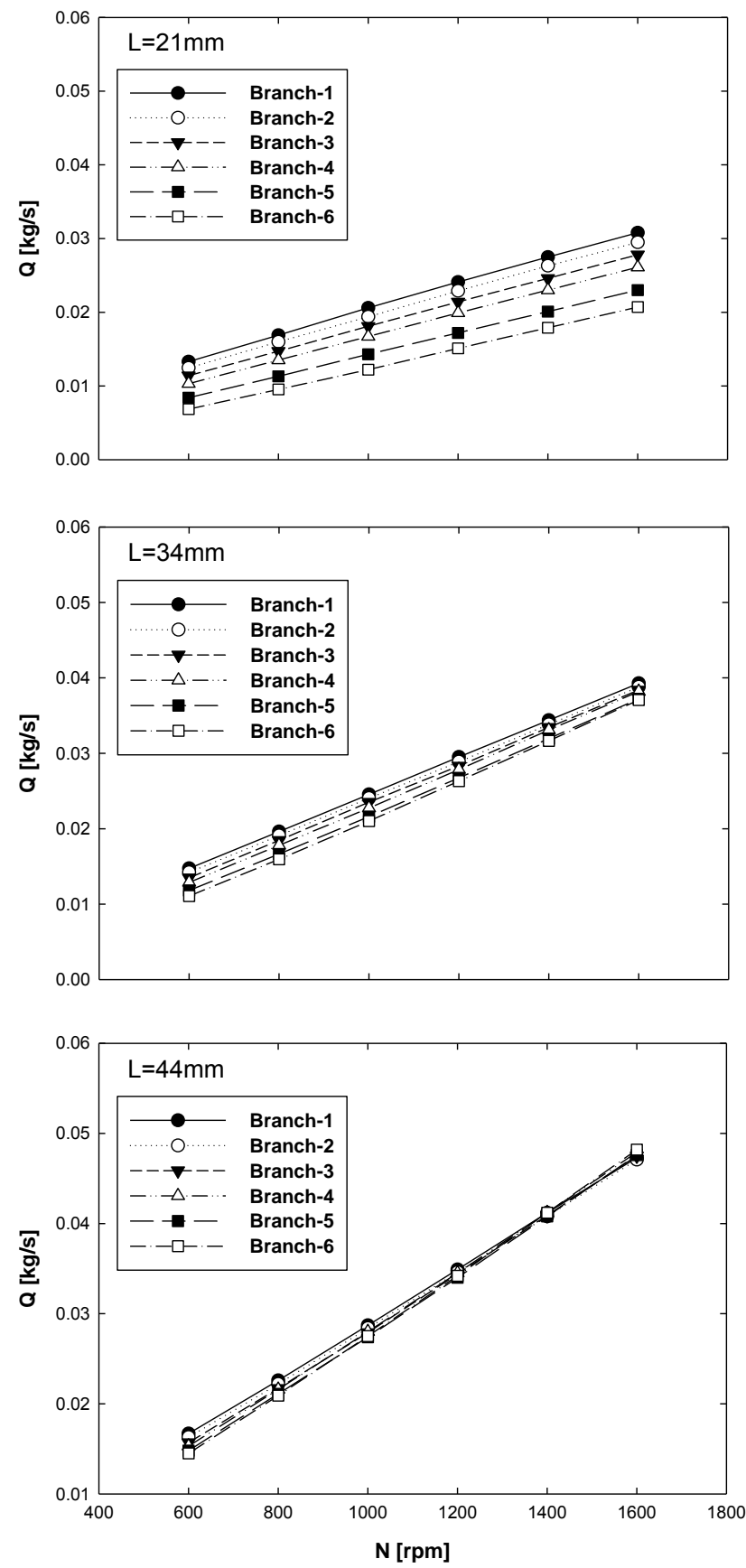

Fig. 8 Comparison of mass flow rate according to engine speed
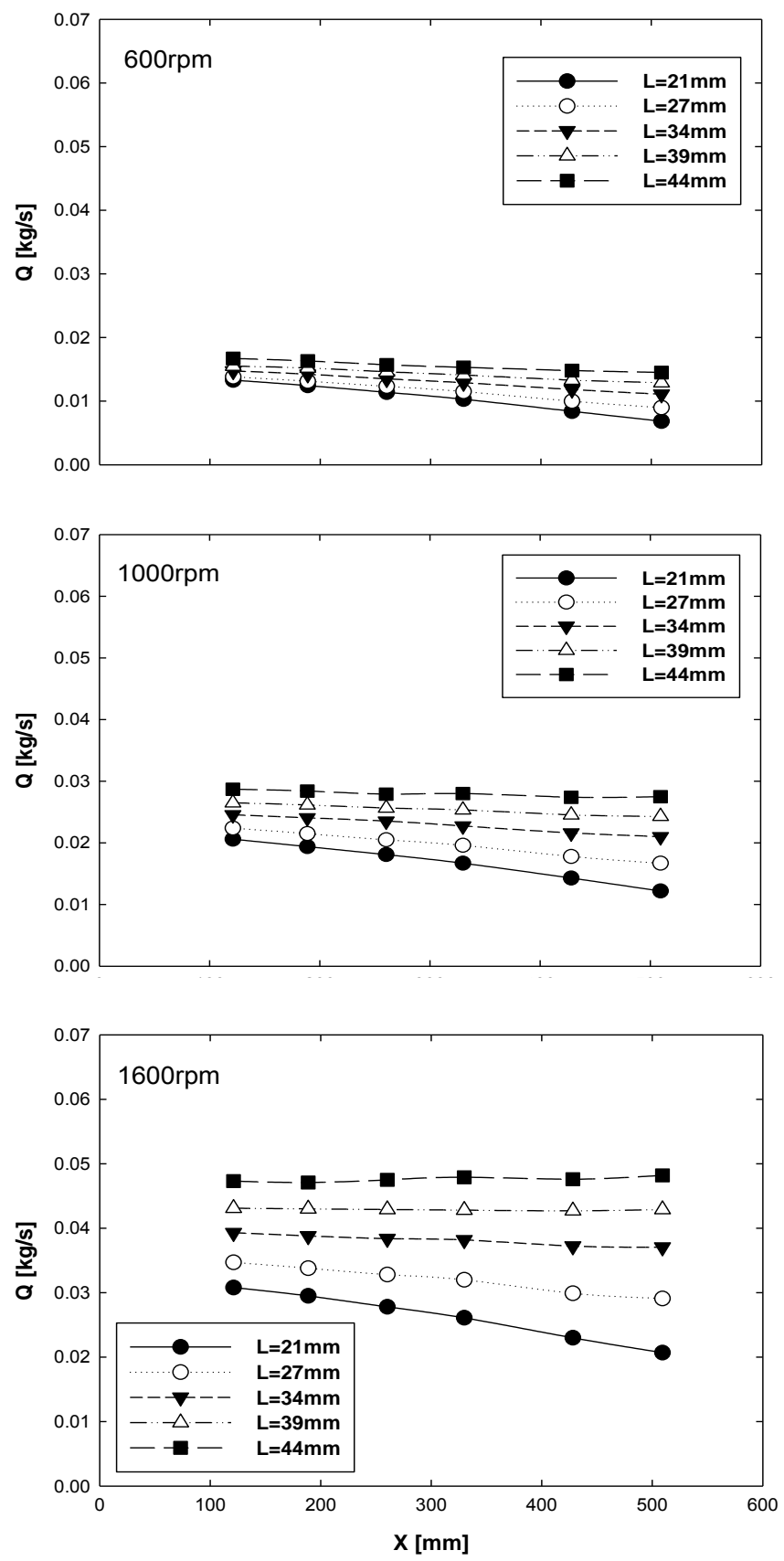

Fig. 9 Comparison of mass flow rate according to branch distance

In the lubrication performance of the transmission, although the transmission fluid is equally supplied from the main shaft, the discharging mass flow rate of transmission fluid is strongly influenced by the branch distance from the inlet as well as the length of branch.

The uniformity of mass flow rate is reduced with increasing of engine speed. The mass flow rate increases with engine speed as shown in Fig. 8. As shown in Fig. 8, the discharging mass flow rate of transmission fluid is higher in the shaft length of $44 \mathrm{~mm}$ than the shaft length of $21 \mathrm{~mm}$. It can be also found the discharging mass flow rate of transmission fluid slowly increases with the increasing of engine rotating rate. Also, as the branch length is longer, the change of discharging mass flow rate at each branch is lower, and the change of discharging 
mass flow rate is reduced as the increasing of the engine rotating number.

Fig.9 represents the variation of discharging mass flow rate with the change of branch distance from the inlet. The increasing rate of the discharging mass flow rate with branch length is higher at the engine rotating rate of 1600 rpm than 600rpm. On the other hand, as the length of branch become shorter, the discharging mass flow rate is decreased in order of the branches.

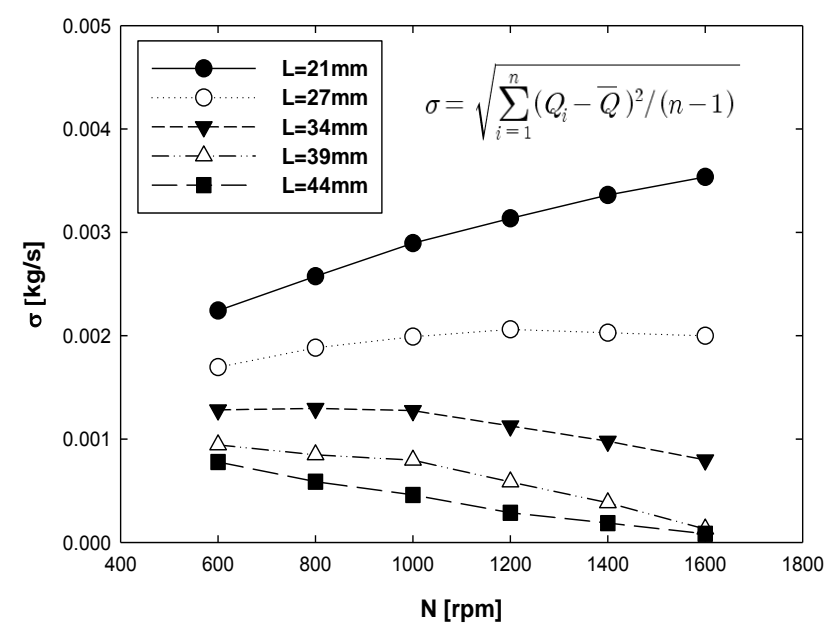

Fig. 10 Distribution of standard deviation at branch

Finally, the characteristic of the discharging mass flow rate with the length of the branch and the engine rotating rate was calculated from Eq. (10) and can be shown in Fig. 10. As a result, it is found that as the length of the branch is shorter, the deviation of the discharging mass flow rate become larger. These behaviours arise from the stronger centrifugal force with the long length of the branch.

$$
\sigma=\sqrt{\sum_{i=1}^{n}\left(Q_{i}-\bar{Q}\right)^{2} /(n-1)}
$$

\section{Conclusion}

Numerical simulation has been conducted to analyze the flow characteristics of lubrication system of commercial vehicle transmission. From numerical simulation, it can be concluded that the discharging mass flow rate is largely influenced by the length of the branch of transmission main shaft and engine speed.

This numerical simulation shows that the discharging mass flow rate from each branch tends to rise when the engine speed is high. Also as the length of branch is long, the discharging mass flow rate is increased.

\section{Acknowledgement}

This research was financially supported by the second stage BK21 Project, the Ministry of Education, Science Technology (MEST) and Korea Institute for Advancement of Technology (KIAT) through the Human Resource Training Project for Regional Innovation.

\section{References}

[1] Junsu Park, Haksu Kim, Duckjea Lee, Siyoul Jang and Kwansoo Han, "A Study on the Transmission Fluid Flow in the Main Shaft of the Automatic Transmission", Proceeding of the 2006 KSAE Spring Annual Meeting, pp.2427-2432, 2006.

[2] Junsu Park, Daehwan Oh, Duckjae Lee, Haksu Kim, Siyoul Jang, Kwansoo Han and Jin-Sung Kim, "A study on the Lubrication Behaviors of Automatic Transmission Fluid inside the Main Shaft through the Oil Holes by Fluent", Proceeding of the 2007 KSAE Spring Annual Meeting, pp.547-552, 2007.

[3] Dong Hoon Park, Tae Seok Seo, Do Gi Lim, Hee Bock Cho, "Theoretical Investigation on Automatic Transmission Efficiency", SAE, pp.49-62, 1996.

[4] Michael A Kluger, Douglas R. Fussner, Bob Roethler, “ A Performance Comparison of Various Automatic Transmission Pumping Systems", SAE, pp.33-40, 1996.

[5] Sangmyung Chun, Younghwan Park, Siyoul Jang, "A Study on Effects of Aerated Engine Oil on Engine Lubrication System by Changing Lubrication Related Parts", KASE Fall Annual Meeting, pp.433-438, 2000

[6] Seungyong Yang, .Sungjong Cha, "Simulation of Cavitating Flow in a Gerotor Oil Pump", KASE Fall Annual Meeting, pp.599-604, 2005

[7] Jiang, Y and Perng, C.Y., "An Efficient 3D Transient Computational Model for Vane Oil Pump and Gerotor Oil Pump Simulations." SAE-970841, pp.91 100, 1997. 